\title{
Distributed 3D Navigation and Collision Avoidance for Nonholonomic Aircraft-like Vehicles
}

\author{
Giannis P. Roussos, Dimos V. Dimarogonas, Kostas J. Kyriakopoulos
}

\begin{abstract}
This paper builds upon previous work of the authors to present a methodology for the decentralized control of multiple 3-dimensional nonholonomic vehicles, utilizing Navigation Functions. The kinematic, non-holonomic, 3-dimensional model considered is chosen to resemble the motion of an aircraft by preventing any movement along the lateral or perpendicular axis, as well as avoiding high yaw rotation rates. The discontinuous feedback control law used is based on the artificial potential field generated by a Dipolar Navigation Function and steers the agents away from each other and towards their destinations, while respecting the non-holonomic constraints present. The performance of the proposed control strategy is formally guaranteed and verified by non-trivial simulation results.
\end{abstract}

\section{INTRODUCTION}

Nonholonomic systems [1] are of great interest in the control community since they apply to a number of real world paradigms, e.g. wheeled mobile robots, Autonomous Underwater Vehicles (AUVs) and Unmanned Aerial Vehicles (UAVs), or automated Air Traffic Control (ATC) in general. In such applications stabilization to a goal configuration, along with collision avoidance with static obstacles or other agents operating in the same area, is required.

As shown in [2], nonholonomic systems cannot be stabilized by any time invariant, smooth state feedback controller, requiring either a time varying or a discontinuous controller. Astolfi [3], Canudas de Wit et. al. [4] and Bloch et. al. [5] have proposed control schemes for the stabilization of a single non-holonomic vehicle using a discontinuous control law, although no collision avoidance strategy has been incorporated. Approaches that additionally perform obstacle avoidance have been proposed that make use of Navigation Functions [6] by Lopes and Koditschek [7], and Tanner et. al. [8]. Team control theory has also been used for the navigation of mobile nonholonomic robots [9], where intermediate waypoints are derived via optimization and a simple controller is used between them. Navigation Functions have been successfully used to control multiagent systems consisting of a number of nonholonomic agents in centralized [10] and decentralized schemes [11]. These approaches address 2dimensional problems, like ground vehicles or aircraft flying at a fixed altitude level. In applications that are inherently 3-dimensional, like aircraft flying in 3-dimensional space or

Giannis P. Roussos and Kostas J. Kyriakopoulos are with the Control Systems Lab, Department of Mechanical Engineering, National Technical University of Athens, 9 Heroon Polytechniou Street, Zografou 15780, Greece. $\{$ jrous, kkyria\}@mail.ntua.gr

D. V. Dimarogonas is with the Laboratory for Information and Decision Systems, Massachusetts Institute of Technology, Cambridge, MA, U.S.A. ddimar@mit.edu underwater vehicles, the above solutions cannot be directly applied as the extension to 3 dimensions is not trivial and requires the assumption of an augmented motion model, compliant with the real system's kinematic constraints.

Previous work on the control of 3D nonholonomic agents include approaches by Aicardi et al. based on a velocity vector field [12], [13] and tracking of a 2D path that has been expanded empirically to $3 \mathrm{D}$ space [14]. It should be noted though that in these approaches no obstacle avoidance method is used, while the bank angle of the vehicle is not controlled. An approach including obstacle avoidance for a single agent has been proposed by the authors in [15].

This paper presents a novel method for the distributed control of multiple 3-dimensional nonholonomic spherical agents using a kinematic controller, in combination with Dipolar Navigation Functions [8]. The nonholonomic model used for the agents is chosen to represent aircraft flying in 3-dimensional space, as it takes into account the kinematic constraints on the lateral and perpendicular motion that apply on the aircraft. Furthermore, the control law is more intuitive and less conservative than previous Navigation Function based controllers [16], while being engineered to keep the yaw rotation rate minimum [15], as it is common for a conventional fixed-wing aircraft. This control strategy forces the agents to follow feasible nonholonomic trajectories that avoid collisions with each other or the workspace boundary, and lead to the desired configuration. Being a reactive, realtime method, this approach is robust with respect to modeling or measurement errors; any deviation from the desired path is directly taken into account by our approach.

The rest of the paper is organized as follows: section II describes the nonholonomic model used for the agents and the problem treated, followed by section III, where the Dipolar Navigation Functions framework used in this paper is presented. In section IV, the proposed feedback control scheme is introduced and analyzed, while section $\mathrm{V}$ includes computer simulation that support the derived results. The conclusions of this paper are summarized in section VI.

\section{SYSTEM AND PROBLEM DEFINITION}

Each agent $i$ is described by a 3-dimensional kinematic nonholonomic model. The state $\mathbf{n}_{i}$ of agent $i, i=1, \ldots, N$ consists of its position vector $\mathbf{n}_{i 1}$ and orientation $\mathbf{n}_{i 2}$ :

$$
\mathbf{n}_{i}=\left[\begin{array}{c}
\mathbf{n}_{i 1} \\
\mathbf{n}_{i 2}
\end{array}\right], \quad \mathbf{n}_{i 1}=\left[\begin{array}{c}
x_{i} \\
y_{i} \\
z_{i}
\end{array}\right], \quad \mathbf{n}_{i 2}=\left[\begin{array}{c}
\phi_{i 1} \\
\phi_{i 2} \\
\phi_{i 3}
\end{array}\right]
$$




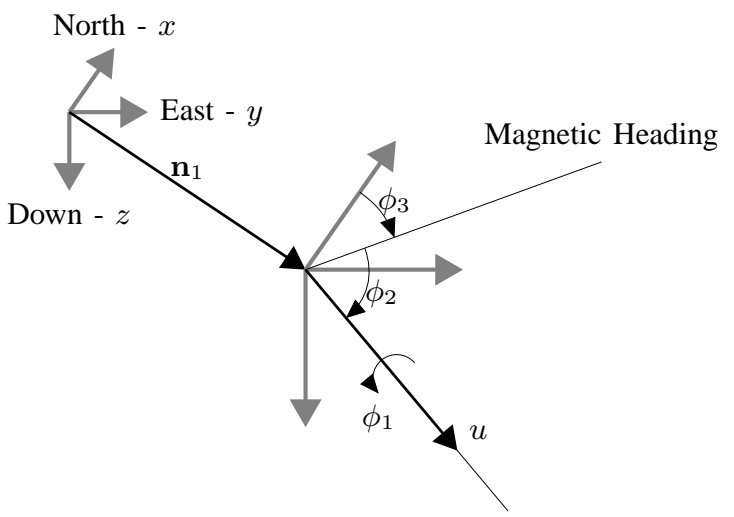

Fig. 1: Earth-Fixed Coordinates

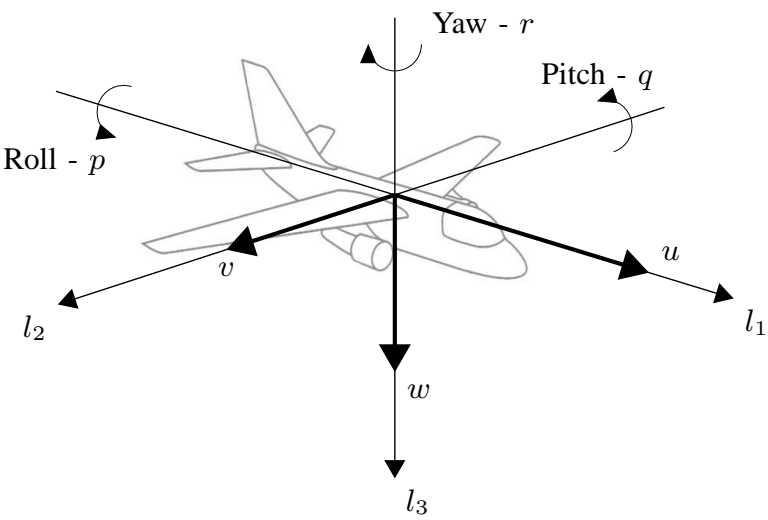

Fig. 2: Body-Fixed Coordinates

where $\left[\begin{array}{lll}\phi_{i 1} & \phi_{i 2} & \phi_{i 3}\end{array}\right]^{T}$ are $x y z$ Euler angles. Let this Earth-fixed coordinate system follow the NED (North-EastDown) convention with $x_{i}$ pointing North, $y_{i}$ East, and $z_{i}$ Down. Consequently $\phi_{i 1}, \phi_{i 2}, \phi_{i 3}$ express bank, elevation and azimuth angles of agents $i$ respectively, as shown in Figure 1 . Let us now define the 3 body-fixed axes $l_{i 1}, l_{i 2}$ and $l_{i 3}$, with $l_{i 1}$ pointing forward, $l_{i 2}$ to the right and $l_{i 3}$ downwards with respect to agent $i$, as shown in Figure 2. The input $\mathbf{v}_{i}$ of each agent consists of the body-fixed, longitudinal velocity $u_{i}$ (along axis $l_{i 1}$ ) and the 3 earth-fixed angular velocities $\omega_{i k}=\dot{\phi}_{i k}, k=1,2,3$ :

$$
\mathbf{v}_{i}=\left[\begin{array}{llll}
u_{i} & \omega_{i 1} & \omega_{i 2} & \omega_{i 3}
\end{array}\right]^{T}
$$

This selection of inputs resembles well the motion of an aircraft, as it does not allow any motion along the bodyfixed lateral $l_{2}$ or perpendicular $l_{3}$ axes. The kinematics of the system described above are:

$$
\dot{\mathbf{n}}_{i}=\mathbf{R}_{i} \cdot \mathbf{v}_{i}
$$

where $\mathbf{R}_{i}=\mathbf{R}\left(\mathbf{n}_{i 2}\right) \in \mathbb{R}^{6 \times 4}$ is a transformation matrix [17]:

$$
\mathbf{R}_{i}=\left[\begin{array}{cc}
\mathbf{J}_{i} & \mathbf{0}_{3 \times 3} \\
\mathbf{0}_{3 \times 1} & \mathbf{I}_{3}
\end{array}\right], \quad \mathbf{J}_{i}=\mathbf{J}\left(\mathbf{n}_{i 2}\right)=\left[\begin{array}{c}
c \phi_{i 3} c \phi_{i 2} \\
s \phi_{i 3} c \phi_{i 2} \\
-s \phi_{i 2}
\end{array}\right]
$$

The choice of this extended 3D unicycle as a high level model for the aircraft is considered adequate for the short- term motion planning task that we consider in this paper. It is assumed that a lower level controller, like the Flight Management System (FMS) or the autopilot will be onboard to realize the trajectories provided by our control scheme.

\section{A. Problem Statement}

The problem under consideration in this paper is to design a control law for each of $N$ spherical agents of radius $r_{i}$ and state $\mathbf{n}_{i}$, described by the kinematic model (1), that will steer each agent $i$ via the inputs: $u_{i}, \omega_{i 1}, \omega_{i 2}, \omega_{i 3}$ to its desired position and direction (elevation and azimuth), $\mathbf{n}_{i 1 d}$ and $\phi_{i 2 d}, \phi_{i 3 d}$ respectively, while avoiding collisions with each other or the boundary $\vartheta W$ of the given workspace $W \subset \mathbb{R}^{3}$. Each agent is assumed to have knowledge of the position, the orientation and the longitudinal velocity of all other agents, but not of their destinations. Finally, the workspace is assumed to be spherical of radius $r_{\text {world }}$.

The scenario described above resembles the case of Air Traffic Management (ATM), where each aircraft can monitor the position, orientation and velocity of neighboring aircraft through surveillance, but has no knowledge of the destinations other than its own. The fact that the method is fully 3D means that each aircraft can use vertical as well as horizontal maneuvering to exploit the available airspace and stay away from conflicts. As the decentralization of Air Traffic Control is thought to be a solution to the increasing air traffic load, the control scheme that follows can be useful in the design of future ATM systems. Other application where such an algorithm may be considered are multiple Autonomous Underwater Vehicles (AUVs) or Unmanned Aerial Vehicles (UAVs) operating in the same area.

\section{Dipolar Navigation Functions}

Navigation Functions are not suitable for the control of a non-holonomic agent, as they do not take into account the kinematic constraints that apply on such a vehicle. Use of the original Navigation Function, as introduced by Koditschek and Rimon in [6], with a feedback law for the control of a nonholonomic agent can lead to undesired behavior, like having the agent rotate in place. In order to overcome this difficulty, Dipolar Navigation Functions have been developed [10], that offer a significant advantage: the integral lines of the resulting potential field are all tangent to the target orientation at the origin, eliminating the need for in-place rotation, as the agent is driven to the origin with the desired orientation. This is achieved by considering the plane whose normal vector is parallel to the desired orientation, and includes the origin, as an additional artificial obstacle $H_{n h}$.

The Navigation Function used in this paper is:

$$
\Phi_{i}=\frac{\gamma_{d i}+f_{i}}{\left(\left(\gamma_{d i}+f_{i}\right)^{k}+H_{n h_{i}} \cdot G_{i} \cdot \beta_{0_{i}}\right)^{1 / k}}
$$

which is constructed as explained in detail in [16].

The function $G_{i}$ is a measure of proximity to collisions involving vehicle $i: G_{i}$ is zero when the $i-t h$ vehicle participates in a conflict, i.e. the sphere occupied by agent $i$ intersects with other agents' spheres, and takes positive 
values away from any conflicts, while $\gamma_{d i}=\left\|\mathbf{n}_{i 1}-\mathbf{n}_{i 1 d}\right\|^{2}$, with $\|\cdot\|$ denoting the euclidean norm, is the squared distance of agent $i$ from its destination $\mathbf{n}_{1 i d}$. The function $f_{i}=$ $f_{i}\left(G_{i}\right)$ is necessary in a distributed approach as it is used in proximity situations in order to ensure that $\Phi_{i}$ attains positive values even when agent $i$ has reached its destination. Thus agent $i$ can be temporarily driven away from its destination in order to facilitate the convergence of neighboring agents.

As the workspace is considered spherical with radius $r_{\text {world }}$, the workspace bounding obstacle is $\beta_{0 i}=r_{\text {world }}^{2}-$ $\left(\left\|\mathbf{n}_{i 1}\right\|+r_{i}\right)^{2}$. The reader is referred to [16] for more details on the construction of $G_{i}, f_{i}$.

The factor $H_{n h i}$ renders the potential field dipolar. As explained before it is responsible for the repulsive potential created by the artificial obstacle used to align the trajectories at the destination with the desired orientation $n_{2 d}$ :

$$
\begin{aligned}
H_{n h i} & =\epsilon_{n h}+n_{n h i} \\
n_{n h i} & =\left\|J_{i d}^{T} \cdot\left(n_{i 1}-n_{i 1 d}\right)\right\|^{2} \\
J_{i d} & =J\left(n_{i 2 d}\right)
\end{aligned}
$$

where $\epsilon_{n h}$ is a small positive constant.

Finally, $k$ is a positive tuning parameter for this class of Navigation Functions. The potential function given above has been used in [11] and has proven navigation properties, i.e., it provides almost global convergence to the destination, along with guaranteed collision avoidance. A simple 2-D example of a Dipolar Navigation Function is presented in Figure 3, depicting the field in the simple case where no obstacles are present. It can been seen that the surface $x=0$ divides the workspace of radius $r_{\text {world }}=100$ in two parts, and forces all the integral lines (crossing the isocurves at a right angle) to approach the target $(0,0)$ parallel to the $y$ axis.

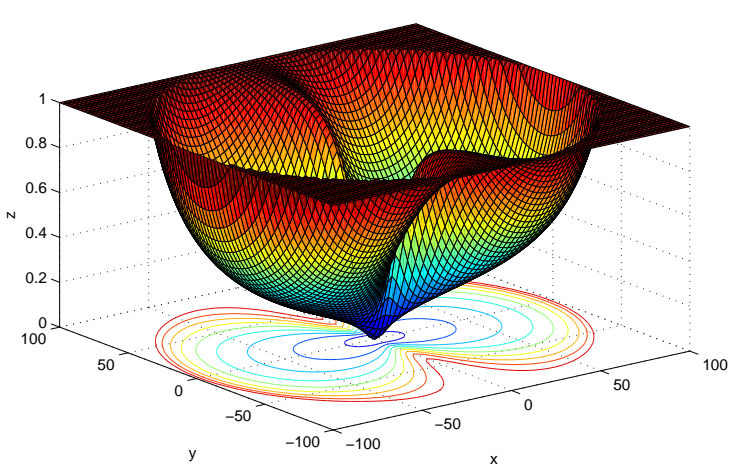

Fig. 3: 2-D Dipolar Navigation Function

\section{3D Non-HolONOMIC NAVIGATION}

\section{A. Control Law}

The proposed control law for agent $i, i=1, \ldots, N$ is as follows:

$$
\begin{aligned}
u_{i} & =-\operatorname{sgn}\left(P_{i}\right) \cdot F_{i}-\left(\frac{\partial \Phi_{i}}{\partial t}+\left|\frac{\partial \Phi_{i}}{\partial t}\right|\right) \frac{1}{2 P_{i}} \\
\omega_{i 1} & =-k_{\phi_{i 1}}\left(\phi_{i 1}-\phi_{\mathbf{n h} i 1}\right) \\
\omega_{i k} & =-k_{\phi_{i k}}\left(\phi_{i k}-\phi_{\mathbf{n h} i k}\right)+\dot{\phi}_{\mathbf{n h} i k}, \quad k=2,3
\end{aligned}
$$

where

$$
\begin{aligned}
F_{i} & =k_{u} \cdot\left\|\nabla_{i} \Phi_{i}\right\|^{2}+k_{z} \cdot\left\|\mathbf{n}_{i 1}-\mathbf{n}_{i 1 d}\right\|^{2} \\
P_{i} & =\mathbf{J}_{i}^{T} \cdot \nabla_{i} \Phi_{i} \\
\nabla_{i} \Phi_{j} & =\frac{\partial \Phi_{j}}{\partial \mathbf{n}_{i 1}} \\
\frac{\partial \Phi_{i}}{\partial t} & =\sum_{j \neq i} u_{j} \nabla_{j} \Phi_{i}^{T} \cdot \mathbf{J}_{j}
\end{aligned}
$$

with $\Phi_{i}=\Phi_{i}\left(n_{i 1}\right)$ being the above Dipolar Navigation Function (2), and $k_{u}, k_{z}, k_{\phi_{i k}}$ positive real gains. The angles $\phi_{\mathbf{n h} i k}$ are defined as follows:

$$
\begin{aligned}
& \phi_{\mathbf{n h} i 3} \triangleq \operatorname{atan} 2\left(\operatorname{sgn}\left(p_{i}\right) \Phi_{i y}, \operatorname{sgn}\left(p_{i}\right) \Phi_{i x}\right) \\
& \phi_{\mathbf{n h} i 2} \triangleq \operatorname{atan} 2\left(-\operatorname{sgn}\left(p_{i}\right) \Phi_{i z}, \sqrt{\Phi_{i x}^{2}+\Phi_{i y}^{2}}\right) \\
& \phi_{\mathbf{n h} i 1} \triangleq \operatorname{atan} 2\left(\operatorname{sgn}\left(p_{i}\right) c \phi_{2} \cdot \omega_{3}, \operatorname{sgn}\left(p_{i}\right) \omega_{2}\right)
\end{aligned}
$$

where $\Phi_{i x}=\frac{\partial \Phi_{i}}{\partial x_{i}}, \Phi_{i y}=\frac{\partial \Phi_{i}}{\partial y_{i}}, \Phi_{i z}=\frac{\partial \Phi_{i}}{\partial z_{i}}$, and $p_{i}=\mathbf{J}_{i d}^{T}$. $\left(\mathbf{n}_{i 1}-\mathbf{n}_{i 1 d}\right)$ is the current position vector with respect to the destination, projected on the longitudinal axis of the desired orientation $\left(l_{i 1 d}\right)$. The functions $\operatorname{sgn}$ and atan 2 are:

$$
\begin{gathered}
\operatorname{sgn}(x) \triangleq \begin{cases}1, & \text { if } x \geq 0 \\
-1, & \text { if } x<0\end{cases} \\
\operatorname{atan} 2(y, x) \triangleq \arg (x, y), \quad(x, y) \in \mathbb{C}
\end{gathered}
$$

Thus $\operatorname{sgn}\left(p_{i}\right)$ is equal to 1 in front of the target configuration and -1 behind it. In order to ensure continuity of the above angles on the destination (where the gradient vector is zero) we use the following approximation scheme [18]:

$$
\hat{\phi}_{\mathbf{n h} i k} \triangleq \begin{cases}\phi_{\mathbf{n h} i k}, & \rho_{k}>\epsilon \\ \frac{\phi_{\mathbf{n h} i k}\left(-2 \rho_{k}^{3}+3 \epsilon \rho_{k}^{2}\right)+\phi_{i k d}\left(-2\left(\epsilon-\rho_{k}\right)^{3}+3 \epsilon\left(\epsilon-\rho_{k}\right)^{2}\right)}{\epsilon^{3}}, & \rho_{k} \leq \epsilon\end{cases}
$$

for $k=1,2,3$, where $\rho_{1}=\sqrt{c \phi_{2}^{2} \cdot \omega_{3}^{2}+\omega_{2}^{2}}, \rho_{2}=\left\|\nabla_{i} \Phi_{i}\right\|$ and $\rho_{3}=\sqrt{\Phi_{i x}^{2}+\Phi_{i y}^{2}}$. Thus the angles $\hat{\phi}_{\mathbf{n h} i k}$ are continuous when $\rho_{k}=0$ as $\lim _{\rho_{k} \rightarrow 0} \hat{\phi}_{\mathbf{n h} i k}=\left.\hat{\phi}_{\mathbf{n h} i k}\right|_{\rho_{k}=0}=\phi_{i k d}, k=$ $1,2,3$. Consequently whenever $\mathbf{n}_{i 1}=\mathbf{n}_{i 1 d}$, i.e., agent $i$ is at its target position, we have:

$$
\hat{\phi}_{\mathbf{n h} i k}=\phi_{i k d}, \quad k=1,2,3
$$

As can be seen in the control law for the longitudinal velocity (3a), the term $-\left(\frac{\partial \Phi_{i}}{\partial t}+\left|\frac{\partial \Phi_{i}}{\partial t}\right|\right) \frac{1}{2 P_{i}}=$ $-\max \left(\frac{\partial \Phi_{i}}{\partial t}, 0\right) \cdot \frac{1}{P_{i}}$ is zero whenever the partial derivative $\frac{\partial \Phi_{i}}{\partial t}$ is non-positive, while the term is activated when $\frac{\partial \Phi_{i}}{\partial t}>$ 0 . As $\frac{\partial \Phi_{i}}{\partial t}$ sums the effect of all but the $i-t h$ agent on $\Phi_{i}$, the condition $\frac{\partial \Phi_{i}}{\partial t}>0$ implies that the motion of all other agents tends to increase $\Phi_{i}$, and therefore agent $i$ must take that into account to cancel the increase rate and ensure that its Navigation Function decreases over time. In the contrary when $\frac{\partial \Phi_{i}}{\partial t} \leq 0$ the term $-\frac{1}{2 P_{i}}$ is not used as it is not required. This modification of the control law makes sure that the term $-\frac{1}{2 P_{i}}$ is used only when it is absolutely necessary, thus making the control law less conservative compared to previous approaches [19], that always include such a term, 
as well as more intuitive. The importance of the above will be made clear in the stability analysis that follows.

\section{B. Stability Analysis}

Theorem 1: Each agent $i$ described by model (1) under the control law (3) is asymptotically stabilized to its target $\mathbf{n}_{i 1 d}, \quad \phi_{i 2 d}, \quad \phi_{i 3 d}$.

Proof: As the control scheme is discontinuous, we will use Lyapunov analysis for nonsmooth systems to prove the stability of the system under the control law (3). The following Lyapunov function candidate is used:

$$
V=\sum_{i} V_{i}, \quad V_{i}=\Phi_{i}+\frac{1}{2} \sum_{k=2}^{3}\left(\phi_{i}-\phi_{\mathbf{n h} i k}\right)^{2}
$$

The generalized derivative of $V$ [20] is:

$$
\partial V=\left[\begin{array}{c}
\sum_{i} \nabla_{1} \Phi_{i} \\
\vdots \\
\sum_{i} \dot{\nabla}_{N} \Phi_{i} \\
1 / 2 \nabla_{\phi_{12}}\left(\phi_{12}-\phi_{\mathbf{n h} 12}\right)^{2} \\
1 / 2 \nabla_{\phi_{13}}\left(\phi_{13}-\phi_{\mathbf{n h} 13}\right)^{2} \\
\vdots \\
1 / 2 \nabla_{\phi_{N 2}}\left(\phi_{N 2}-\phi_{\mathbf{n h} N 2}\right)^{2} \\
1 / 2 \nabla_{\phi_{N 3}}\left(\phi_{N 3}-\phi_{\mathbf{n h} 33}\right)^{2} \\
1 / 2 \nabla_{\phi_{\mathbf{n h} 12}}\left(\phi_{12}-\phi_{\mathbf{n h} 12}\right)^{2} \\
1 / 2 \nabla_{\phi_{\mathbf{n h} 13}}\left(\phi_{13}-\phi_{\mathbf{n h} 13}\right)^{2} \\
\vdots \\
1 / 2 \nabla_{\phi_{\mathbf{n h N 2}}\left(\phi_{N 2}-\phi_{\mathbf{n h} 2}\right)^{2}} \\
1 / 2 \nabla_{\phi_{\mathbf{n h} 3}}\left(\phi_{N 3}-\phi_{\mathbf{n h} N 3}\right)^{2}
\end{array}\right]=\left[\begin{array}{c}
\sum_{i} \nabla_{1} \Phi_{i} \\
\vdots \\
\sum_{i} \dot{\nabla}_{N} \Phi_{i} \\
\left(\phi_{12}-\phi_{\mathbf{n h} 12}\right) \\
\left(\phi_{13}-\phi_{\mathbf{n h} 13}\right) \\
\vdots \\
\left(\phi_{N 2}-\phi_{\mathbf{n h} N 2}\right) \\
\left(\phi_{N 3}-\phi_{\mathbf{n h} 33}\right) \\
-\left(\phi_{12}-\phi_{\mathbf{n h} 12}\right) \\
-\left(\phi_{13}-\phi_{\mathbf{n h} 13}\right) \\
\vdots \\
-\left(\phi_{N 2}-\phi_{\mathbf{n h} 22}\right) \\
-\left(\phi_{N 3}-\phi_{\mathbf{n h} N 3}\right)
\end{array}\right]
$$

Let us then consider the multi-agent system $\dot{\mathbf{x}}=f(\mathbf{x})$ resulting from the composition of (1):

$$
\mathbf{x}=\left[\begin{array}{c}
\mathbf{n}_{11} \\
\vdots \\
\mathbf{n}_{N 1} \\
\phi_{12} \\
\phi_{13} \\
\vdots \\
\phi_{N 2} \\
\phi_{N 3} \\
\phi_{\mathbf{n h 1 2}} \\
\phi_{\mathbf{n h 1 3}} \\
\vdots \\
\phi_{\mathbf{n h} N 2} \\
\phi_{\mathbf{n h} N 3}
\end{array}\right], \quad f(\mathbf{x})=\left[\begin{array}{c}
u_{1} \mathbf{J}_{1} \\
\vdots \\
u_{N} \mathbf{J}_{N} \\
\omega_{12} \\
\omega_{13} \\
\vdots \\
\omega_{N 2} \\
\omega_{N 3} \\
\dot{\phi}_{\mathbf{n h} 12} \\
\dot{\phi}_{\mathbf{n h} 13} \\
\vdots \\
\dot{\phi}_{\mathbf{n h} 22} \\
\dot{\phi}_{\mathbf{n h} N 3}
\end{array}\right]
$$

The Filippov set [21] of the above system is:

$$
K[f]=\left[\begin{array}{c}
K\left[u_{1}\right] \mathbf{J}_{1} \\
\vdots \\
K\left[u_{N}\right] \mathbf{J}_{N} \\
\omega_{12} \\
\omega_{13} \\
\vdots \\
\omega_{N 2} \\
\omega_{N 3} \\
\dot{\phi}_{\mathbf{n h} 12} \\
\dot{\phi}_{\mathbf{n h} 13} \\
\vdots \\
\dot{\phi}_{\mathbf{n h} 2} \\
\dot{\phi}_{\mathbf{n h} N 3}
\end{array}\right]
$$

By the control law (3a) we deduce:

$$
K\left[u_{i}\right]=K\left[-\operatorname{sgn}\left(P_{i}\right)\right] \cdot F_{i}-\left(\frac{\partial \Phi_{i}}{\partial t}+\left|\frac{\partial \Phi_{i}}{\partial t}\right|\right) \frac{1}{2 P_{i}}
$$

Using the chain rule given in [22] we can calculate the generalized time derivative of $V$ as follows:

$$
\begin{aligned}
\dot{\tilde{V}}= & \bigcap_{\xi \in \partial V} \xi^{T} K[f]= \\
= & \sum_{i} \sum_{j} K\left[u_{i}\right] \nabla_{i} \Phi_{j}^{T} \mathbf{J}_{i}+ \\
& +\sum_{i} \sum_{k=2}^{3}\left(\phi_{i k}-\phi_{\mathbf{n h}_{i k}}\right)\left(\omega_{i k}-\dot{\phi}_{\mathbf{n h}_{i k}}\right)= \\
\stackrel{(7)}{=} & \sum_{i} K\left[u_{i}\right] \nabla_{i} \Phi_{i}^{T} \mathbf{J}_{i}+\sum_{i} \sum_{j \neq i} K\left[u_{j}\right] \nabla_{j} \Phi_{i}^{T} \mathbf{J}_{j}- \\
& -\sum_{i} \sum_{k=2}^{3} k_{\phi_{i k}}\left(\phi_{i k}-\phi_{\mathbf{n h} i k}\right)^{2}= \\
= & \sum_{i}\left\{K\left[-s g n\left(P_{i}\right)\right] \cdot P_{i} F_{i}-\frac{1}{2}\left(\frac{\partial \Phi_{i}}{\partial t}+\left|\frac{\partial \Phi_{i}}{\partial t}\right|\right)\right\}+ \\
& +\sum_{i} \frac{\partial \Phi_{i}}{\partial t}-\sum_{i} \sum_{k=2}^{3} k_{\phi_{i k}}\left(\phi_{i k}-\phi_{\mathbf{n h} i k}\right)^{2}= \\
= & \sum_{i}\left\{-\left|P_{i}\right| F_{i}-\frac{1}{2}\left(\left|\frac{\partial \Phi_{i}}{\partial t}\right|-\frac{\partial \Phi_{i}}{\partial t}\right)\right\}- \\
& -\sum_{i} \sum_{k=2}^{3} k_{\phi_{i k}}\left(\phi_{i k}-\phi_{\mathbf{n h} i k}\right)^{2} \leq 0
\end{aligned}
$$

Since each $V_{i}$ and consequently $V$ is regular [20] and the level sets of $V$ are compact, the nonsmooth version of LaSalle's invariance principle [22] can be applied. We can thus conclude that the trajectory of the closed-loop system converges to the largest invariant subset $S$ :

$$
\begin{aligned}
& S \triangleq\{\mathbf{n} \mid 0 \in \dot{\tilde{V}}\}= \\
&=\left\{\mathbf{n}:\left(-\left|P_{i}\right| F_{i}-\frac{1}{2}\left(\left|\frac{\partial \Phi_{i}}{\partial t}\right|-\frac{\partial \Phi_{i}}{\partial t}\right)=0 \forall i\right) \wedge\right. \\
&\left.\quad\left(\phi_{i k}=\phi_{\mathbf{n h}_{i k}} \forall i, k=2,3\right)\right\}
\end{aligned}
$$

Thus inside $S$ we have $P_{i}=0$ or $F_{i}=0$. The condition $F_{i}=0$ holds only when $\mathbf{n}_{i 1}=\mathbf{n}_{i 1 d}$, i.e., when each agent has reached its target position with no other agents are close enough to make $f_{i}>0$, while $P_{i}=0$ holds at the target and whenever the agent's $i$ longitudinal axis is normal to the field's gradient $\nabla_{i} \Phi_{i}$. In the later case though, if the agent is away from the destination, i.e. $F_{i} \neq$ 0 , at least one of the elevation and azimuth angles $\phi_{i 2}$ and $\phi_{i 3}$ are not equal to $\phi_{\mathbf{n h} i 2}$ and $\phi_{\mathbf{n h} i 3}$ respectively, and therefore the corresponding configurations are outside $S$. As a result only the target position is included in $S$. Moreover, by (5) and the condition $\phi_{i k}=\phi_{\mathbf{n h}_{i k}} \forall i, k=$ 2,3 , we deduce that the set $S$ reduces to the singleton $\left\{\mathbf{n}:\left(\mathbf{n}_{i 1}=\mathbf{n}_{i 1 d} \forall i\right) \wedge\left(\phi_{i k}=\phi_{i k d} \forall i, k=2,3\right)\right\}$, i.e., all the 
agents are stabilized to their destinations with the desired elevation and azimuth angles.

Remark: From the control law (3a) we can see that the linear velocity tends to infinite values when $P_{i} \rightarrow 0$, i.e., when the projection of the field's gradient on the agent's longitudinal axis is very small. This is the case when the gradient vector is normal to the agent's longitudinal axis: $\nabla_{i} \Phi_{i} \perp l_{i 1}$. In this case at least one of the angles $\phi_{i k}, k=$ 2,3 will be not equal to the the corresponding $\phi_{\mathbf{n h} i k}$, and therefore $\left(\phi_{i k}-\phi_{\mathbf{n h} i k}\right)$ is non zero for at least one of $k=$ 2,3 . Calculating the dynamics of this term we have:

$$
\frac{d}{d t}\left(\phi_{i k}-\phi_{\mathbf{n h} i k}\right)=-k_{\phi_{i k}}\left(\phi_{i k}-\phi_{\mathbf{n h} i k}\right)+\dot{\phi}_{\mathbf{n h} i k}-\dot{\phi}_{\mathbf{n h} i k}
$$

As a result the absolute value $\left|\phi_{i k}-\phi_{\mathbf{n h} i k}\right|$ is always decreasing in time and each term $\phi_{i k}-\phi_{\mathbf{n h} i k}, k=2,3$ is stabilized to 0 . Thus if the absolute angle between the field's gradient and $l_{i 1}$ is initaly smaller than $\frac{\pi}{2}$, it will always remain in $\left[0, \frac{\pi}{2}\right)$ and the set $G \triangleq\left\{\mathbf{n} \mid \exists i: \nabla_{i} \Phi_{i} \perp l_{i 1}\right\}$, where $P_{i} \rightarrow 0$, will never be reached. Essentially it is required that

$$
P_{i} \cdot p_{i}>0
$$

at the initial conditions, i.e. agents starting in the subspace behind their targets ( where $p_{i}<0$ ) must have the initial negated gradient vector driving them forward $\left(P_{i}<0\right)$, while agents starting in front of their target $\left(p_{i}>0\right)$ must have the negated gradient initially driving them backward $\left(P_{i}>0\right)$. To enforce additionally only forward (or backward) motion, we have to ensure that all agents start in the subspace behind (or in front) of their targets. These mild requirements should not pose practical difficulties in Air Traffic applications, as they represent reasonable physical conditions.

\section{Simulation}

In order to demonstrate the effectiveness of the above control strategy, a computer simulation is presented below. The test case considered consists of 4 agents of radius $r_{i}=0.05, i=1, \ldots, 4$ operating in a spherical workspace of $r_{\text {world }}=1$. The initial positions are spanned near the boundary of the workspace facing inward and the target configurations have been set across the center of the workspace, so that the straight line paths between each start position and the corresponding destination create multi-agent conflicts near the center $(0,0,0)$. Specifically the initial configurations of the agents are:

$$
\begin{aligned}
& \mathbf{n}_{\text {1init }}=\left[\begin{array}{rrrrrrr}
-0.9 & 0 & 0.3 & 0 & 0 & 0 & ]^{T}
\end{array}\right.
\end{aligned}
$$

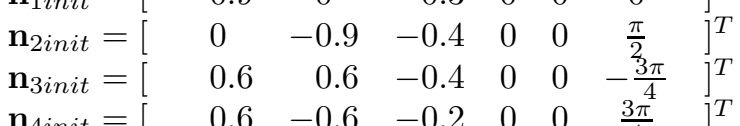

and the target positions and elevation, azimuth angles :

$$
\begin{array}{rrrl}
\mathbf{n}_{11 d}=\left[\begin{array}{rrr}
.9 & 0 & -.3
\end{array}\right]^{T}, & \phi_{12 d}=0, & \phi_{13 d}=0 \\
\mathbf{n}_{21 d}=\left[\begin{array}{rrrl}
0 & .9 & .2
\end{array}\right]^{T}, & \phi_{22 d}=0, & \phi_{23 d}=\frac{\pi}{2} \\
\mathbf{n}_{31 d}=\left[\begin{array}{rrr}
-.6 & -.6 & .2
\end{array}\right]^{T}, & \phi_{32 d}=0, & \phi_{33 d}=-\frac{3 \pi}{4} \\
\mathbf{n}_{41 d}=\left[\begin{array}{rrr}
-.6 & .6 & .4
\end{array}\right]^{T}, & \phi_{42 d}=0, & \phi_{43 d}=\frac{3 \pi}{4}
\end{array}
$$

The results can be seen in Figures 4, 5 and 6 where the trajectories of the agents are plotted as seen from 3 different
Fig. 4

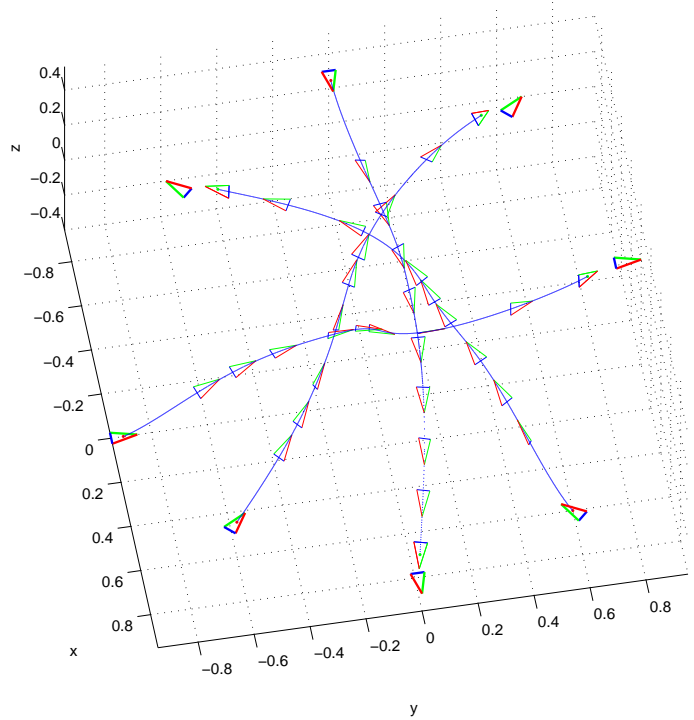

Fig. 5

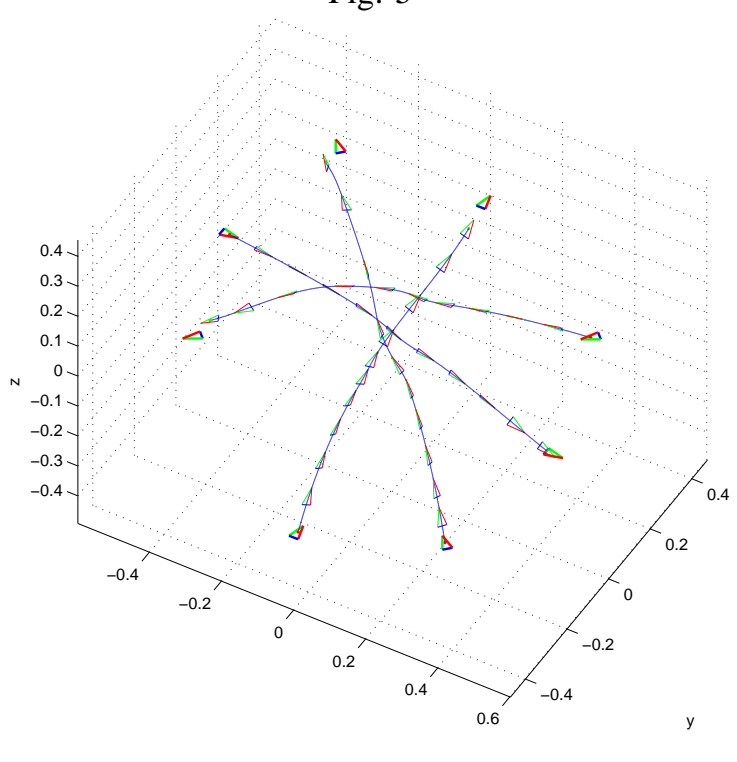

viewing angles. The distances between any two agents are plotted in Figure 7 (solid lines) along with the minimum safety clearance $2 \cdot r_{i}=0.1$.

As the figures demonstrate, the agents follow feasible, nonholonomic 3-dimensional paths avoiding collisions with each other, and converge towards their destinations and directions as intended. Because of the exponential convergence rate, some deviation between the final positions (achieved within the simulation duration) and the desired ones can be observed, although in general the agents approach their destinations with favorable orientations. The distance between any two agents is always higher than the safety margin as no collisions occur. 
Fig. 6
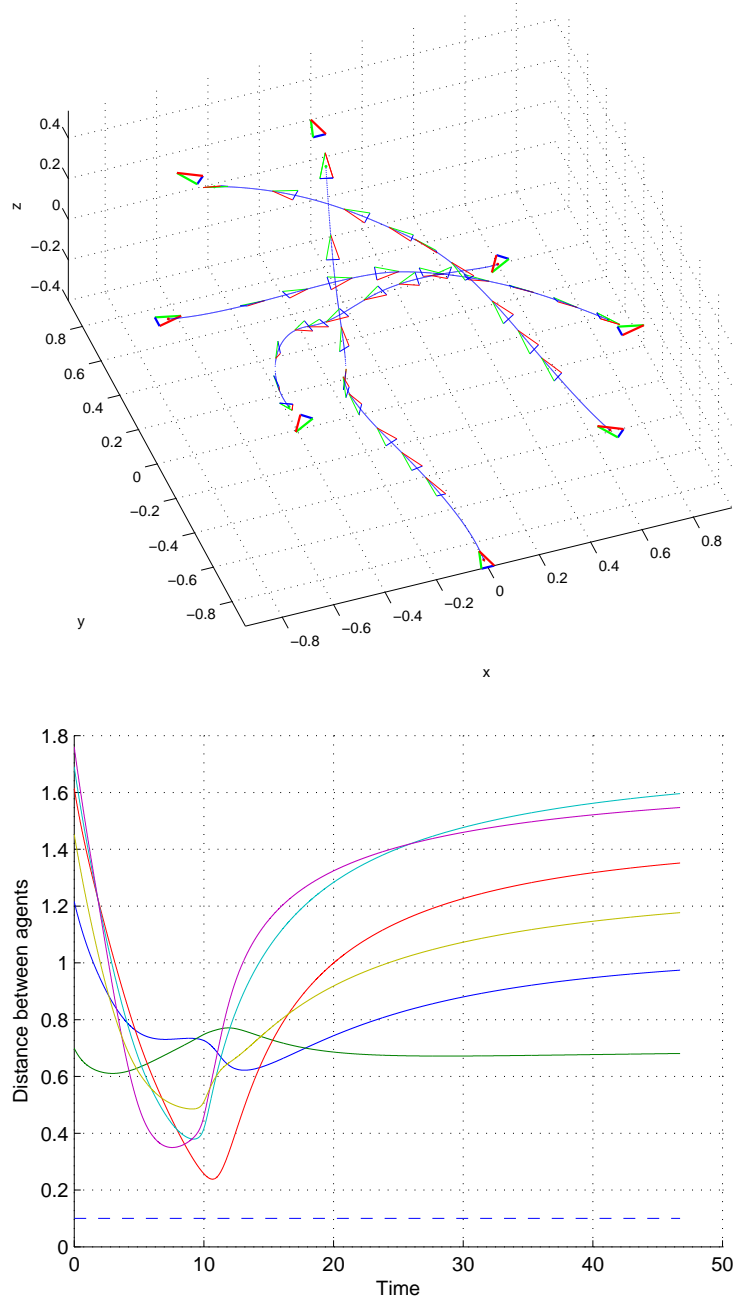

Fig. 7: Distances between agents (solid lines) and minimum safety clearance (dashed line)

\section{CONCLUSIONS}

This paper proposes a Navigation Function based control strategy for multiple 3-dimensional non-holonomic aircraftlike agents. The proposed distributed control scheme steers the agents towards their targets and away from collisions with each other. The discontinuous control law generates trajectories that respect the nonholonomic constraints and the low yaw capability of typical aircraft. The feedback control law makes the control strategy robust with respect to measurement and modeling errors, while the use of Navigation Functions provides guaranteed global convergence and collision avoidance. Our future work is directed towards the better control of the algorithms convergence rate, and thus the agents' velocities.

\section{ACKNOWLEDGEMENTS}

The first and third author of this paper want to acknowledge the contribution of the European Commission through project iFLY.

\section{REFERENCES}

[1] A. M. Bloch, Nonholonomic Mechanics and Control. Springer-Verlag NY, 2003.

[2] R. W. Brockett, "Asymptotic stability and feedback stabilization," in Differential Geometrics in Control Theory (R. S. M. Robert W. Brockett and H. J. Sussmann, eds.), vol. 27 of Progress in Mathematics, pp. 181-191, Boston: Birkhauser Publishers, 1983.

[3] A. Astolfi, "Exponential stabilization of a wheeled mobile robot via discontinuous control," ASME Journal of Dynamic Systems Measurement and Control, vol. 121, pp. 121-125, 1999.

[4] C. Canudas-de-Wit and O. J. Sordalen, "Exponential stabilization of mobile robots with nonholonomics constraints," IEEE Transaction on Automatic Control, vol. 37, no. 11, 1992.

[5] A. M. Bloch, S. V. Drakunov, and M. K. Kinyon, "Stabilization of nonholonomic systems using isospectral flows," SIAM Journal on Control and Optimization, vol. 38, no. 3, pp. 855-874, 2000.

[6] E. Rimon and D. E. Koditschek, "Exact robot navigation using artificial potential functions," IEEE Transactions on Robotics and Automation, vol. 8, no. 5, pp. 501-508, 1992.

[7] G. A. D. Lopes and D. E. Koditschek, "Navigation functions for dynamical, nonholonomically constrained mechanical systems," in Advances in Robot Control (S. Kawamura and M. Svinin, eds.), pp. 135-155, Springer, 2006

[8] H. G. Tanner and K. J. Kyriakopoulos, "Nonholonomic motion planning for mobile manipulators," Proceedings of the 2000 IEEE International Conference on Robotics \& Automation, pp. 1233-1238, 2000.

[9] M. Aicadi and M. Baglietto, "Decentralized supervisory control of a set of mobile robots," European Control Conference 2001, 2001.

[10] H. G. Tanner, S. Loizou, and K. J. Kyriakopoulos, "Nonholonomic navigation and control of cooperating mobile manipulators," IEEE Transactions on Robotics and Automation, vol. 19, no. 1, pp. 53-64, 2003.

[11] D. V. Dimarogonas and K. J. Kyriakopoulos, "A feedback control scheme for multiple independent dynamic non-point agents," International Journal of Control, vol. 79, no. 12, pp. 1613-1623, 2006.

[12] M. Aicardi, G. Cannata, G. Casalino, and G. Indiveri, "Guidance of 3D underwater non-holonomic vehicle via projection on holonomic solutions," Symposium on Underwater Robotic Technology, SURT 2000, June 2000.

[13] M. Aicardi, G. Casalino, and G. Indiveri, "Closed loop control of 3D underactuated vehicles via velocity field tracking," Advanced Intelligent Mechatronics, 2001. Proceedings. 2001 IEEE/ASME International Conference on, vol. 1, pp. 355-360, 2001.

[14] G. Ambrosino, M. Ariola, U. Ciniglio, F. Corraro, A. Pironti, and M. Virgilio, "Algorithms for 3D UAV path generation and tracking," 2006 45th IEEE Conference on Decision and Control, pp. 5275-5280, 2006.

[15] G. P. Roussos, D. V. Dimarogonas, and K. J. Kyriakopoulos, "3D navigation and collision avoidance for a non-holonomic vehicle," 2008 American Control Conference, Seattle, Washington, USA, 2008.

[16] D. V. Dimarogonas, S. G. Loizou, K. J. Kyriakopoulos, and M. M. Zavlanos, "A feedback stabilization and collision avoidance scheme for multiple independent non-point agents," Automatica, vol. 42, no. 2, pp. 229-243, 2006.

[17] T. I. Fossen, Guidance and Control of Ocean Vehicles. John Wiley \& Sons, 1994.

[18] M. Egerstedt and X. Hu, "Formation constrained multi-agent control," Robotics and Automation, IEEE Transactions on, vol. 17, pp. 947-951, Dec 2001.

[19] D. V. Dimarogonas and K. J. Kyriakopoulos, "A feedback stabilization and collision avoidance scheme for multiple independent nonholonomic non-point agents," Proceeding of the 2005 IEEE International Symposium on Intelligent Control, 2005.

[20] F. Clarke, Optimization and Nonsmooth Analysis. Addison-Wesley, 1983.

[21] A. Filippov, Differential equations with discontinuous right-hand sides. Kluwer Academic Publishers, 1998.

[22] D. Shevitz and B. Paden, "Lyapunov stability theory of nonsmooth systems," IEEE Transactions on Automatic Control, vol. 39, no. 9, pp. 1910-1914, 1994. 